\title{
Implications of growing electronic commerce for freight transportation: A case study of the United States
}

\author{
Minyoung Park*, Jung Ung Min ${ }^{* *}$ and Sang-Yoon Lee ${ }^{* * *}$
}

\begin{abstract}
Recent advancements in information and communication technologies have led to the rapid growth of electronic commerce market. In the United States, e-commerce retail sales for 2002 reached \$45.6 billion, indicating an increase of 26.9\% from 2001 while total retail sales increased $3.1 \%$ during the same period. Although e-commerce sales account for only $1.4 \%$ of total sales in this country, forecasts show that the magnitude of digital economy will continue to expand. The logistical requirements of e-commerce goods that extend to each customer's address stimulate greater complexity in traditional supply chain management, potentially causing higher costs for freight supply chain participants. To harness the economic potential of e-commerce, it is important to encourage the development of a freight transportation system that will support its steady growth, while avoiding the possible negative effects from the changes in freight transportation. Due to the intrinsic nature of e-commerce goods, advances in home delivery have the potential to promote the growth of e-commerce as well as to create sustainable urban freight transportation systems. Based on the case study of the United States, this paper presents an in-depth discussion of the key challenges arising in home delivery operations, and proposes potential solution strategies that will lead to more efficient and reliable home delivery systems.
\end{abstract}

Keywords: Digital Economic; Electronic Commerce; Package delivery; Home delivery; Urban Freight transpiration.

Submission Date: 11/20/2006 Acceptance Date: 12/7/2006

*Corresponding Author: Professor, Graduate School of Logistics Inha University 253 Yonghyun-dong, Nam-gu, Incheon, 402-751, Korea; tel.+82-32-860-8237, Email: mypark@inha.ac.kr

*** Professor, Graduate School of Logistics, Inha University

${ }^{* * * *}$ Professor, Graduate School of Logistics, Inha University 


\section{Introduction}

Recent advancements in information and communication technologies have led to the rapid growth of electronic commerce market. Despite the recent economic downturn, e-commerce retail sales for 2002 reached $\$ 45.6$ billion in the United States (US), indicating an increase of $26.9 \%$ from 2001 while total retail sales increased 3.1\% during the same period (Census Bureau, 2003). Although e-commerce sales account for only $1.4 \%$ of total sales in this country, forecasts show that the digital economy will continue to grow. To harness the economic potential of e-commerce, therefore, it is important to encourage the development of a freight transportation system that will support the steady growth of digital economy, while avoiding the possible negative effects on society from these changes in freight transportation.

Electronic commerce enables businesses to sell their products and services directly to each individual consumer without establishing a physical point of sale. While some products can be delivered digitally to households (for example, newspaper, airline ticket and music), most products purchased online ultimately must be transported in the physical world. In the e-commerce environment, an efficient and reliable delivery system is essential for gaining customer loyalty and thus obtaining profitability. Consequently, home delivery is increasingly becoming a key element in the supply chain of e-commerce goods. The logistical requirements of e-commerce goods that extend to each customer's address may stimulate greater complexity in existing supply chain management, causing higher costs for freight supply chain participants, including producers, carriers, retailers, and consumers. For example, increase in time-sensitive goods requires the increased number of delivery vehicles. More frequent home-based local deliveries are likely to worsen traffic congestion and environmental problems. This could be even serious especially in urban areas where traffic congestion is already a big concern, making it more difficult for carriers to meet customer demands. Such changes do not pose challenges only for the freight community, but also for transportation agencies that construct, operate, and maintain the transportation system in their control.

Over the past several years, many researchers have investigated the likely impacts of online shopping on transportation, including the movement of freight (Gould, 1998; Golob and Regan, 2001; Lyons, 2002). Although home delivery already plays a crucial role in the distribution chain of goods regardless of being purchased either online or at a physical store, limited attention has been paid to the issues associated with the home delivery in the literature. A recent exception is the work of Cairns (1996), which identified the key issues in grocery home delivery through a series of interviews with representatives from 35 organizations, and proposed some schemes for foods home delivery that may be successfully implemented. Campbell and Salvelsberg (2002) also provide a good example that examined consumer direct service problems faced by individual retailers providing home delivery. This study points to the necessity of integrating order capture (acceptance) and delivery. In more general studies, Nagarajan et al. (2000) and Golob and Regan (2001) have contributed to a comprehensive understanding of the potential impacts of information technology (IT) on freight transportation, addressing structural changes in the freight and logistics industry generated by e-commerce. Recently, a conceptual framework to evaluate the impacts of 
IT and e-commerce on urban logistics system has been proposed by Nemoto et al. (2001). Their study identifies the major stakeholders and examines their concerns related to urban logistics systems.

Considering the growing importance of home delivery for e-commerce, we present in this paper an in-depth discussion of the logistical challenges emerging in home delivery operations through a case study of the United States. Recognizing key issues related to home delivery has important implications for developing potential solution strategies for the issues, and thus should be the first step towards developing more efficient and reliable home delivery systems. Advances in home delivery will eventually help to promote e-commerce as well as to create sustainable urban freight transportation systems.

This paper is organized as follows. In the next section, we briefly examine the relationship between e-commerce and home delivery based on relevant statistics. This is followed by an investigation of the operational characteristics and historical development of home delivery services with a particular example of the United States. Key issues arising in the real world home delivery operations of e-commerce goods are then identified, which provide implications for developing innovative strategies that will enhance home delivery efficiency and eventually facilitate e-commerce. Finally, the paper concludes by summarizing the findings of this study and presenting future research directions

\section{Electronic commerce and home delivery}

A distinctive aspect of the digital economy is its ability to expedite information exchange and improve information accessibility for products, markets and customers. As indicated in Gould and Golob(1998), while consumers frequently use the Internet to gather information about products, many are still purchasing off-line for the reason that online shopping eliminates the experiential aspect and recreational advantage of shopping.

However, there is an emerging tendency for people to view goods at a physical shop and then buy them online at lower prices, resulting in the development of a hybrid model that combines the strengths of traditional store-based retail and pure e-commerce retail (Gulati and Garino, 2000). The recent trend in traditional retail market is moving towards the use of all possible marketing options including mail, e-commerce and physical stores. A good example is the Office Depot that has recently integrated its Web site and its existing catalog sales and physical stores operations to form a single, seamless retailing network. In this example, the Internet is just another channel that buttresses other two channels (www.officedepot.com). From the perspective of customers, the integrated channels make shopping simpler and more convenient since the customers can research their choices online, clicking through rich information on features and prices. Once the customers find the best model for their needs, they can buy it online and have it delivered to their home. In case they need the product immediately, they can check the site to ensure its stock at their neighborhood Office Depot store and go pick it up themselves. There are several important reasons consumers choose to purchase goods online, including at-home convenience, 24 hour $/ 7$ day 
availability, low prices, and available product information. As a result, e-commerce is growing at an annual growth rate of over $25 \%$ and the most recent estimate predicts that more than $10 \%$ of all US retail sales are expected to take place online by 2008 (Johnson et al., 2003).

The growth of e-commerce has impacted many aspects of business practices such as globalization of trade, small batch, customized production and just-in-time distribution, causing a number of changes in the volume and patterns of goods movements. Although the extent to which e-commerce will affect transportation may depend on the types of markets and products, smaller and more frequent shipments become the consequences of e-commerce as manufacturers and distributors communicate directly with consumers. E-commerce customers are increasingly demanding faster transportation with increased service quality. These changes driven by ecommerce bring business opportunities to the logistics service providers, particularly couriers handling parcel delivery, delivering goods to each customer's home quickly and reliably in small batches. A recent survey by Morlok et al. (2000) provides evidence of this tendency, showing that the parcel industry has been the fastest growing major segment of the freight transportation industry in the US for the past several decades (see $<$ Fig. $1>$ ).

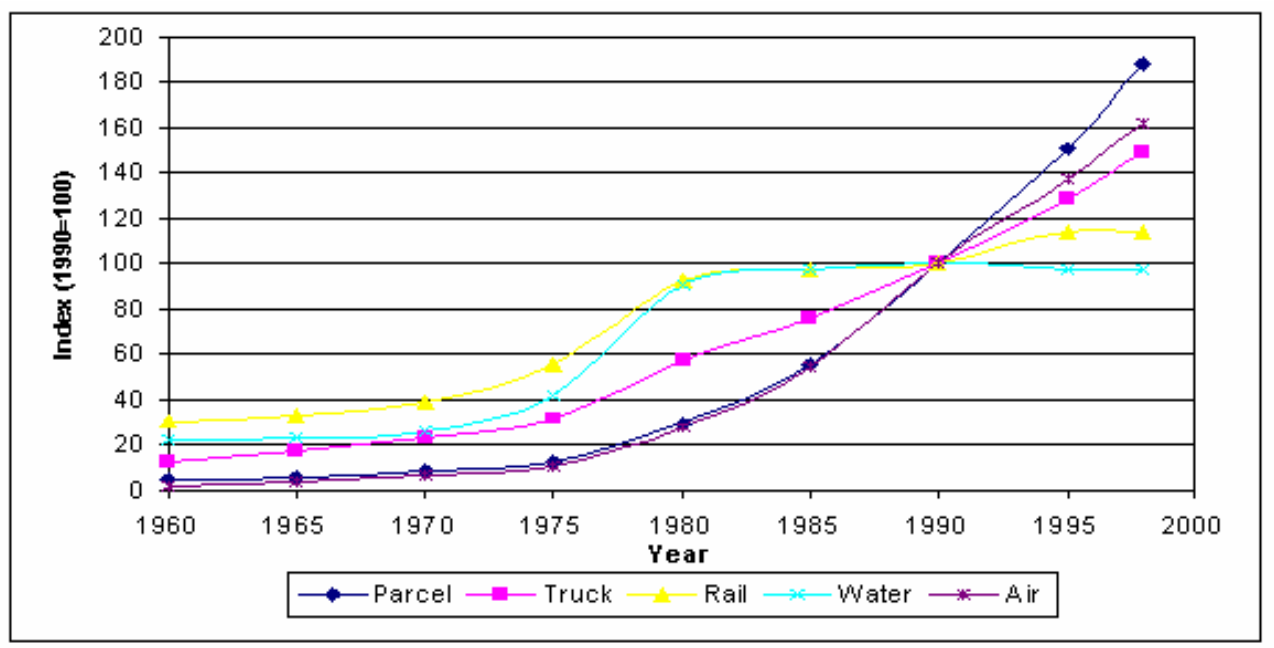

Fig. 1. Growth of the parcel industry in the United States

The most significant impact of e-commerce on freight transportation is the increase in direct home delivery of smaller shipments. An effort to validate the correlation between e-commerce and home delivery with some relevant statistics may be untimely at this point because e-commerce is still at an early stage of development, and thus it is too early to obtain statistically significant data on e-commerce. The early predictions were clearly exaggerated, but it does not mean that the impact will not be significant. One of the problems with trying to identify statistics assessing the ecommerce market, particularly with regard to determining trends over time, is that a vast array of different survey results and estimates are available from different sources, making reliability and comparison difficult to judge. Fortunately, national statistics institutes have recently begun to 
encompass e-commerce within their surveys. Since the fourth quarter of 1999, the US Census Bureau (2003) has estimated and reported quarterly US e-commerce retail sales, compared with total retail sales, which provides a good context for conjecturing the scale and growth of ecommerce market. To investigate the linkage between e-commerce and home delivery, we compared the growth rate of the e-commerce retail sales estimated by the US government with that of the UPS domestic package delivery revenues. UPS is a leading company in the parcel industry, accounting for almost half of total revenues in the US parcel industry(UPS, 2003), thereby implying half of total number of home deliveries.

Table 1 presents quarterly growth rates of e-commerce retail sales and UPS domestic package delivery revenues. Using the same data, we draw a graph to illustrate a linkage between ecommerce growth and home delivery growth. Fig.2 explains that they are closely related, indicating that the changes in the growth rates of the UPS domestic revenues reflect to a large extent the changes in the e-commerce retail sales, although there may be many other factors that explain the growth of home delivery market. The fluctuations in both e-commerce retail sales and home delivery are mostly determined by the seasonal factors. Their relationship is more apparent in the UPS Deferred product (within 2 or 3 business days) rather than its total including Overnight service and Ground service (within 6 days for domestic shipments).

\section{Table 1}

Quarterly growth rRates of e-commerce retail sales and UPS domestic package delivery revenues in the US

\begin{tabular}{|c|c|c|c|c|c|c|}
\hline \multirow{3}{*}{$\begin{array}{c}\text { Period } \\
\text { (Quarterly) }\end{array}$} & \multicolumn{2}{|c|}{ E-commerce Retail Sales ${ }^{1)}$} & \multicolumn{4}{|c|}{ UPS Domestic Revenue $^{2)}$} \\
\hline & \multirow{2}{*}{ \$ millions } & \multirow{2}{*}{ Growth Rate (\%) } & \multicolumn{2}{|c|}{ \$ millions } & \multicolumn{2}{|c|}{ Growth Rate (\%) } \\
\hline & & & Deferred & Total & Deferred & Total \\
\hline 1999.4Q & 5,481 & N.A. & 797 & 6,074 & 22.8 & N.A. \\
\hline 2000. 1Q & 5,814 & 6.1 & 694 & 5,841 & -12.9 & -3.8 \\
\hline $2 Q$ & 6,346 & 9.2 & 690 & 5,890 & -0.6 & 0.8 \\
\hline $3 Q$ & 7,266 & 14.5 & 690 & 5,928 & 0.0 & 0.6 \\
\hline $4 Q$ & 9,459 & 30.2 & 836 & 6,343 & 21.2 & 7.0 \\
\hline 2001. 1Q & 8,256 & -12.7 & 716 & 5,976 & -14.4 & -5.8 \\
\hline $2 Q$ & 8,246 & -0.1 & 711 & 5,981 & -0.7 & 0.1 \\
\hline $3 Q$ & 8,236 & -0.1 & 664 & 5,806 & -6.6 & -2.9 \\
\hline $4 Q$ & 11,178 & 35.7 & 802 & 6,234 & 20.8 & 7.4 \\
\hline 2002. 1Q & 9,880 & -11.6 & 700 & 5,903 & -12.7 & -5.3 \\
\hline $2 Q$ & 10,265 & 3.9 & 695 & 5,908 & -0.7 & 0.1 \\
\hline $3 Q$ & 11,061 & 7.8 & 672 & 5,889 & -3.3 & -0.3 \\
\hline $4 Q$ & 14,334 & 29.6 & 801 & 6,224 & 19.2 & 5.7 \\
\hline
\end{tabular}

Source: 1) US Census Bureau (2003)

2) UPS (2003) 


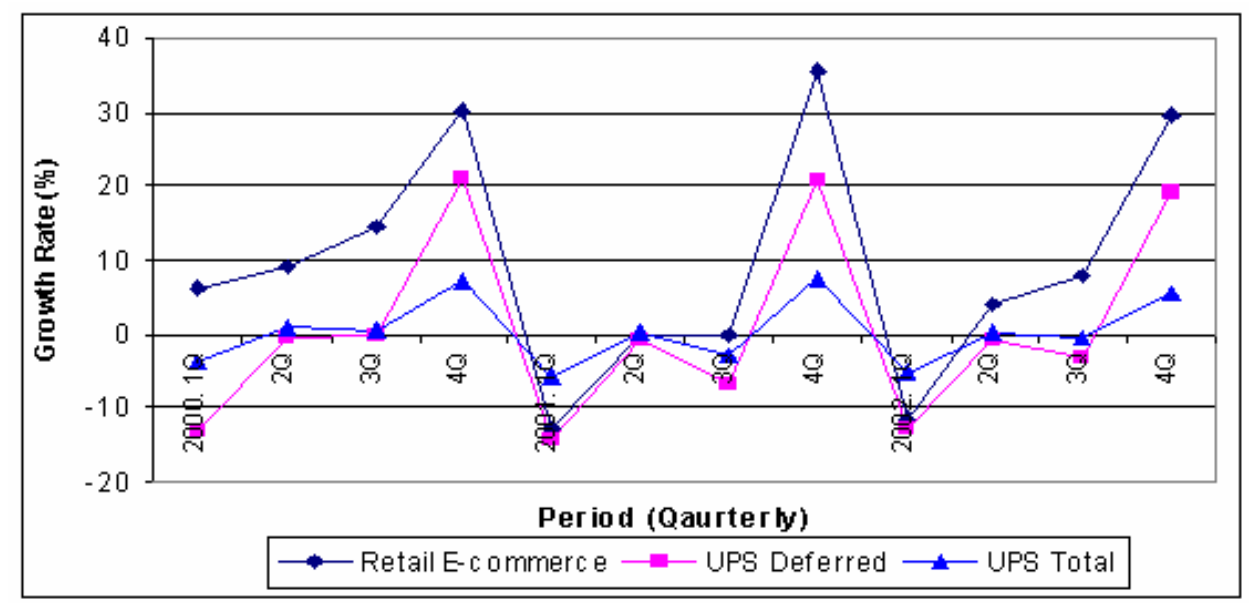

Fig. 2. Relationship between e-commerce and home delivery

\section{The overview of home delivery}

\subsection{Definition and delivery characteristics}

Home delivery is referred to as deliveries of goods to the customers' homes (or another location of the customers' choice such as workplace) rather than customers having to collect the goods inperson from a physical point of sale. In home delivery operations, therefore, the physical distribution of the goods from the point of purchase to the customer needs to be organized and carried out by specialized delivery companies rather than by the customer. Many other situations lead to home delivery, including the delivery of traditional mail (or telephone) order products and the delivery of large and/or heavy items such as furniture. Most e-commerce products involve home delivery, but all home deliveries are not generated by e-commerce. For the simplicity of discussion, we will focus on the home delivery of goods generated by e-commerce, mostly in the context of the home delivery of packaged products (or parcel).

In traditional store-based commerce, goods are typically distributed in sequence from the manufacturer, to the wholesaler, to the retailer, and finally to the customer, although the distribution of goods varies, depending on the type of business. A relatively large share of the distribution of goods occurs through distribution centers which are owned by producers, wholesalers or logistics service providers. From a distribution point of view, retail shops function as the end points of the distribution chain that a delivery carrier involves. The customers mostly have to take care of the "last-mile" transportation of goods, i.e., delivery from the physical point of purchase to home, though in some cases such as large and/or heavy items, the end of distribution chain may be extended to the consumer's households. However, this is not the case with e-commerce. As 
companies and consumers can easily make contact with more potential purchasers and suppliers, ecommerce has changed the shape of traditional supply chain. Products purchased online must be transported from a plant or distribution center directly to customer's home in a timely manner regardless of shipment size. This implies that e-commerce generates a different need for the transportation of goods from traditional delivery practices.

The distribution of goods to retail shops mostly involves the frequent delivery of packaged units, consisting of one or more boxes, pallets or containers, filled with a number of homogeneous goods. In contrast, e-commerce delivery has usually only one (relatively small) item for each address. Even though there may be some level of bundling, it consists of the bundling of very different goods for one region, but the goods are not packaged together. Therefore, delivery of ecommerce goods requires a different service from that of traditional freight transportation.

Table 2 summarizes the distinct characteristics of e-commerce delivery compared to those of traditional delivery

Table 2

Characteristics of e-commerce goods delivery

\begin{tabular}{c|c|c}
\hline Attributes & Traditional Delivery & E-commerce Delivery \\
\hline \hline Distribution Chain & Producer - Wholesaler - Retailer & Online Retailer - Customer \\
\hline Shipment size & Large & Small \\
\hline Shipment type & Homogeneous & Heterogeneous \\
\hline Number of loads (density) & High & Low \\
\hline Number of delivery stops & One or more stops & Many stops \\
\hline Delivery failure & Few & Many \\
\hline Delivery frequency & Low & High \\
\hline Delivery time sensitivity & Low & High \\
\hline Number of vehicle required & Low & Small \\
\hline Vehicle size & Large & High \\
\hline Delivery cost per each load & Small &
\end{tabular}

\subsection{Historical development of home delivery services}

The changes on the demand side lead to developments in supply. Companies that are going to be most successful in the digital economy should be able to deliver their goods timely and reliably. Two recent surveys of trucking firms (Nagarajan, 2000; Golob and Regan, 2002) show that most traditional trucking firms are investing in advanced information technologies in 
order to respond to the customer demands in the forms of just-in-time distribution, package express, door-to-door delivery and same-day service. It is also noteworthy that the freight transportation sector, an industry that has been relatively slow in adopting new technologies and cultures, has shifted its focus to developing strategic alliances with shippers. A good example is a successful relationship between UPS and manufacturers (for example, Dell computer) or online retailers such as eBay.

\section{Existing nationwide home delivery service providers}

Historically, there have been two major nationwide providers of home delivery, United Parcel Service (UPS) and the US Postal Service (USPS). With a national delivery network that has been built up over decades and decades, UPS(2003) has a truly comprehensive network and tremendous volume (roughly 12 million packages per day nationwide) to spread over its network, which results in a highly profitable package delivery business. While UPS is the only private company with such a comprehensive delivery network, there is another entity that has an even more comprehensive network. By law, the USPS (2003) must provide delivery to every address in the US on every business day. However, in contrast to the UPS, the USPS must provide this coverage regardless of profitability. The USPS delivers parcels to the home primarily through its Priority Mail product, which is predominantly a business-to-consumer (B2C) and consumer-to-consumer (C2C) product (roughly 85\%), and it has much lower exposure to the business-to-business (B2B) market than its rival package carriers such as UPS, FedEx, and Airbone Express. The Priority Mail product is not time-definite and does not have full track-and-trace capability yet, but it has lower pricing than UPS products (especially for items less than five pounds in weight). Priority Mail is primarily a two- or three-day product. On the other hand, UPS has almost exactly the opposite mix, with about $80-85 \%$ of its package volume in the B2B category. We believe that most $\mathrm{B} 2 \mathrm{C}$ packages are being delivered by ground(which is significantly cheaper), but for customers who are willing to pay, UPS offers express package delivery.

\section{Transformation of traditional transportation service providers}

Over the past few years, a number of traditional transportation companies have introduced products specifically designed to benefit from the growth in the $\mathrm{B} 2 \mathrm{C}$ e-commerce delivery business. For example, Federal Express (FedEx) and Airborne Express have recently added B2Cfocused delivery services. Launched by FedEx's Ground in March 2000, FedEx Home Delivery product is designed to meet the needs of catalogers and online retailers and offers shippers and customers standard evening and Saturday delivery as well as several premium service options such as day-specific appointments of delivery. For the fiscal year of 2002, FedEx Ground reported a 21 percent increase in average daily package volume, one-third of which was attributed to its home delivery (FedEx, 2003). Airborne Express has partnered with the USPS to create a new kind of service for the home delivery of e-commerce goods, named "airborne@home." It was designed for business-to-residence shippers who desire a secure, cost-effective shipping solution, in which shipments are picked up by Airborne and delivered to the USPS post office closest to the recipient's home. The local post office then delivers directly to the customers. Demand for this service has 
risen slowly but steadily from about 4,000 packages per day at the beginning of 2000 to around 20,000 packages per day at the end of May, 2002 (Airborne Express, 2003). While Airborne Express's product provides some further choices to shippers, it does not actually add capacity to the system in terms of delivery over the last-mile to the home, since it utilizes existing USPS infrastructure for the final delivery.

\section{Emergence of same-day service providers}

Companies selling goods online for home delivery currently offer several different delivery options, each of which has different delivery charges. However, few of these retailers and delivery companies offer their customers the option of timed delivery (either by day or time slot). A recent trend shows that e-commerce products involving quicker delivery, such as grocery delivery, continue to grow slowly but steadily, leading to the emergence of delivery companies specializing in same-day local pickup and delivery. One successful example is Dynamex, a company with same day operations in many major metropolitan areas in the US and Canada (www.dynamex. com). In its same-day service delivery, times are further broken down within the time horizon of a single day. In general, there is a trade-off between the time of delivery and the price, and the unit cost per mile increases as time-in-transit decreases, as shown in Table 3. Accordingly, increase in local express delivery demands may bring about increased productivity and profitability for transportation service providers.

Table 3

Impacts of delivery time and distance on shipping rate

\begin{tabular}{c|c|c|c}
\hline Service Types & Time Guarantee & Basic Rate & Rate per Distance \\
\hline \hline Priority & Within 60-90 minutes & $\$ 15$ & $\$ 1.75 / \mathrm{mile}$ \\
Rush & Within 2-3 hours & $\$ 12$ & $\$ 1.40 / \mathrm{mile}$ \\
Regular & Within 4 hours & $\$ 10$ & $\$ 1.10 / \mathrm{mile}$ \\
\hline
\end{tabular}

Source: www.dynamex.com

\section{Future perspectives}

The continuing growth in e-commerce may eventually support new entrants into the market (though it clearly did not support the new entrants of the past such as Kosmo, Webvan, Sameday and Urbanfetch). The e-commerce delivery products that utilize the USPS for final delivery are taking a lower-risk approach since less capital investment is required. The companies using this strategy are likely to take advantage of the current B2C market growth opportunity. However, it should be noted that for these participants, the lower risk comes at the price of lower potential margins due to the significant cost of paying the USPS for final delivery. We believe that there is a 
desire among shippers for alternatives to UPS and the USPS, so we also expect FedEx's Home Delivery type of products to grow and attain a modest market share level over time in the ecommerce delivery market segment. Two of the web grocery companies that emerged in the late 1990s, Peapod and Webgrocer are still in business, though neither is very large. A new web grocer, Freshdirect entered the New York City market in late 2002. It is possible that if these companies can weather the poor economy and define an appropriate niche market, they will continue to grow at a modest rate.

\section{Key issues and solution strategies in home delivery operations}

The growth of e-commerce has forced online retailers and delivery companies to address many issues about how they organize and operate their home delivery systems and the level of customer service they are aiming to provide. Different products may have different delivery attributes, so an approach made for say, grocery delivery, may not be essentially suitable when considering the delivery of large items. In this section, we delve into fundamental issues related to home delivery that need to be addressed in the e-commerce environment, leading to implications for delivery companies to answer the question of what changes need to occur in freight transportation and logistics to facilitate e-commerce.

\subsection{Unattended delivery problems}

A major factor for the success of home delivery operations is whether there is someone at the customer's home to receive the delivery. Several social and economic factors are leading to homes being empty for longer periods in a day than they used to be. Some of the factors include inflexible working patterns, long commutes, increases in working women, and the growth in single-person households. These result in a relatively high proportion of first time delivery failure, causing higher operating costs for carriers and lower customer satisfaction.

There are some cases such as grocery home delivery(Cairns, 1996), in which prior arrangements for a time window for delivery are made between online retailers and their customers, although even this case may not guarantee that the customer will be at home at the time of delivery. However, this is not the norm for most home deliveries. The reason is that such pre-arrangements of delivery time slots with customers would increase the inflexibility in carriers' fleet operations, leading to an extremely expensive delivery system for both retailers and carriers. If prearranging delivery times and days with customers do not prove attractive to companies delivering ecommerce products, then there is likely to be a growing need for these companies to develop alternative delivery systems and strategies.

When the customer is not at home at the time of delivery, four alternatives for the carrier are conceivable:

- Delivery at a different date or time: this generates extra costs for the carrier, but is currently 
being implemented as a service in the real world.

- Pre-arrangement of delivery time: this would be unattractive for all parties involved, but it can be an additional option for customers willing to pay extra charge for it.

- Establishment of secure reception boxes: such facilities at or around customer's homes exist in some cases such as in postal services, but are very limited in practice.

- Delivery to a local pickup point: from the points such as convenience stores and gas stations available for 24 hours, customers can pick their goods up at any time of day convenient to the customer, or other 'last mile' delivery companies make prearranged deliveries to customers.

As reported in Nemoto et al. (2001), the concept of local pickup points using convenience stores has been already successfully implemented in Japan. Convenience stores open almost all the time in a day, and thus can offer this service both for extra revenue and because it generates more in-store traffic. This type of delivery scheme may be more effective in a densely populated area where the facilities for pickup like convenient stores or gas stations are densely located within walking distance. However, customers' willingness to pickup their shipment at a local point must be examined carefully before implementation. As a way of dealing with the unattended delivery problems, encouraging the customers to choose deliveries to offices instead of their homes could be an alternative. With a growing number of houses empty during the daytime and the standard delivery times between 8 am and $5 \mathrm{pm}$, the difficulties are obvious. There is clearly a need to better understand when and where customers want to receive deliveries, and then meet these needs. Reducing the likelihood of these delivery problems would help to reduce operating costs of home delivery companies and increase customers' satisfaction for B2C-driven home delivery services, consequently smoothing the progress of e-commerce.

\subsection{Increasing demand for faster delivery}

Increasing demand for faster and more reliable delivery is also an issue for retailers and delivery companies. Many people have a belief that online products must be shipped as soon as possible after the order is made. This is usually perceived as important by electronic retailers in order to match the service levels offered by their competitors. Although there are some customer demands for rapid home delivery (as witnessed by the emergence of same-day service provider serving precisely this needs), there is little evidence to show that the majority of customers expect such service or are prepared to pay extra cost for it.

UPS data provide insights into market trends. Table 4 represents the changes in revenues and number of shipments of UPS domestic package delivery operations over the period from 1994 to 2002. UPS services can be classified into three major categories, Overnight service, Deferred service (within 2 or 3 business days), and Ground (within 6 days for domestic shipments). From the table, it is observed that while demands for Overnight service have been growing at the fastest rate of $7.9 \%$ in revenues and $8.4 \%$ in number of shipments annually, the largest portion of UPS operations is still Ground service, showing $66 \%$ in revenue and $83 \%$ in number of shipments. This indicates that customers have widely differing lead-time expectations. 
Table 4

Changes in the UPS domestic package delivery operations

\begin{tabular}{c|c|c|c|c|c|c|c|c}
\hline \multirow{2}{*}{ Year } & \multicolumn{4}{|c|}{ Revenues (\$ millions) } & \multicolumn{3}{c}{ Number of Shipments (millions) } \\
\cline { 2 - 9 } & Overnight & Deferred & Ground & Total & Overnight & Deferred & Ground & Total \\
\hline \hline 1994 & 2,905 & 1,928 & 12,110 & 16,943 & 148.1 & 158.2 & $2,506.5$ & $2,812.8$ \\
\hline 1995 & 3,269 & 2,041 & 12,463 & 17,773 & 169.0 & 181,1 & $2,517.1$ & $2,867.2$ \\
\hline 1996 & 3,734 & 2,207 & 12,940 & 18,881 & 193.0 & 193.8 & $2,543.8$ & $2,930.6$ \\
\hline 1997 & 4,054 & 2,314 & 12,500 & 18,868 & 208.0 & 195.1 & $2,408.8$ & $2,811.9$ \\
\hline 1998 & 4,690 & 2,464 & 13,496 & 20,650 & 238.3 & 198.9 & $2,449.8$ & $2,887.0$ \\
\hline 1999 & 5,240 & 2,694 & 14,379 & 22,313 & 263.9 & 216.4 & $2,544.1$ & $3,024.4$ \\
\hline 2000 & 5,664 & 2,910 & 15,428 & 24,002 & 285.0 & 232.2 & $2,650.2$ & $3,167.4$ \\
\hline 2001 & 5,433 & 2,893 & 15,671 & 23,997 & 283.5 & 232.9 & $2,620.5$ & $3,136.9$ \\
\hline 2002 & 5,349 & 2,868 & 15,707 & 23,924 & 282.2 & 227.3 & $2,568.4$ & $3,077.9$ \\
\hline $\begin{array}{c}\text { Growth } \\
\text { Rate (\%) }\end{array}$ & 7.9 & 5.1 & 3.3 & 4.4 & 8.4 & 4.6 & 0.3 & 1.1 \\
\hline
\end{tabular}

Source: UPS (2003)

The trend towards faster delivery means that express services are likely to become more important in the overall mix of home delivery services. For some products such as computer chips, the cost of holding inventory already outweighs the relatively high cost of a premium shipment. The balance of that equation is slowly moving in favor of express delivery. As delivery time shortens, this can have the effect of reducing the delivery density and the potential for consolidation of deliveries, consequently increasing the operating cost and delivery vehicle trip generation. In our view, the time between taking customer's order and making deliveries will certainly be faster than at present. Nonetheless, if it would be possible to increase the lead-time between ordering and delivery, this may help to improve delivery efficiency by increasing the delivery density and the potential for consolidation of deliveries, while maintaining a certain level of customer service.

\subsection{Growing needs for more efficient and reliable local delivery}

As already noted, the logistical requirement of e-commerce goods that extends the end of the supply chain to each customer's home may generate many problems in local areas, and potentially causes higher costs in carriers' fleet operations than it used to be. This is mainly due to the small size and low density of e-commerce shipments. The reduction in average shipment sizes leads to an increase in the number of truck trips and a reduction in average vehicle sizes, consequently causing an increase in urban traffic. The low density is also problematic since it leads to an increase in the frequency of delivery stops and a growth in average vehicle-mile traveled. More frequent local deliveries, additionally, involves a proliferation of commercial delivery vehicles to residential areas, which will likely be concerns about the social and environmental impacts of delivery 
vehicles in residential areas. The increasing demands for quicker and more reliable delivery are also raising the weight of local delivery operations in the supply chain of e-commerce goods. In both local and long distance delivery services of e-commerce goods, local movements are a very important segment in the whole delivery process because local (typically urban) movements can take up a large segment of the overall delivery time. Some demands driven by e-commerce are extremely sensitive to the time in transit and time of delivery, and the transit and delivery times are determined mostly by the traffic conditions in urban areas.

1997 Commodity Flow Survey data (BTS, 1999) highlight the importance of local operations - shipments transported within less than 100 miles accounted for $67 \%$ of the weight and $40 \%$ of the value of total goods movements. Another example for this trend is a change in new transport vehicle purchases over the past decade, showing an increase of $8.0 \%$ for small (and median) truck less than 14,000 lbs, from 3,606 thousands in 1991 to 7,774 thousands in 2001, while total number of trucks over 14,000 lbs purchased increased 5.4\% over the same period (Eno Transportation Foundation, 2002).

Concentrated densities of people, office sites, residential areas and accompanying high traffic levels characterize urban (especially metropolitan) areas. Multiple players are competing for space, very often at the same places and at the same times. In a 'just-in-time' environment, people and goods need to operate on the same cycle time. While it is often presumed that freight should be delivered on off-peak hours to relieve traffic congestion in an urban area, as a practical matter, this is often not a solution. The reason is that receivers may not be there to receive goods at off-peak hours and cost factors are often prohibitive. Successful solutions will focus on how to derive the most utility out of available capacity. Examples of desirable distribution strategies may include: eliminating redundant delivery attempts, promoting personal deliveries to residential or office sites where package delivery services are already calling, and efficiently locating warehouse sites.

In planning the routes and schedules of commercial fleet in urban areas, particular interest should be paid to the flexibility of on-time delivery constraints affecting changes in routes and schedules, and the potential effectiveness of various policies intended to decrease redundant delivery attempts, consequently mitigate traffic congestion in urban areas. For solutions that can be acceptable for trucking companies in major urban areas, there will be a need of further researches to examine the feasibility of various alternative operation schemes and to assess their effectiveness in the system.

Among various potential strategies, as a way of eliminating redundant delivery attempts, an additional new service option called 'station-to-station service' can be introduced to the existing 'doorto-door delivery' systems. The idea stems from circumstances in which customers cannot take shipment at a time period convenient to the delivering company. The station-to-station service proposed could be a new option for customers. In this strategy, carriers use 24-hour convenient stores or gas stations as local pick-up and delivery stations in their distribution system, which may be viable if appropriate partnerships between the trucking industry and other industries can be established. As an alternative, offices and apartment buildings can be designed or remodeled with secure delivery boxes for the occupants to allow package delivery firms to leave personal shipments with one-stop delivery. Shipments can be delivered to the workplace or the apartment whether the recipient is on site or not. Providing alternative service option to the system will enhance customers' satisfaction and also enable carriers to more flexible operate their vehicles. Eventually such a system will help to alleviate 
traffic congestion in urban areas by eliminating redundant delivery attempts.

\subsection{The return of goods and reverse logistics}

Home delivery of damaged or faulty goods is an additional issue that supply chain partners should note in terms of costs. These costs mainly consist of transportation costs of removing the damaged goods and distributing the replacement goods, and administrative costs of dealing with the problem that may arise from parties disputing the damage claim. This also causes significant customer inconvenience in having to arrange for the return of the goods as well as for the delivery of replacement goods. As shown in the Office Depot's example, there is an effort of some retailers to facilitate the return of online products, in which online customers can return their purchases to any local shop or pickup truck.

Reverse logistics is concerned with managing the movement of goods back to manufacturers, distributors and retailers, because they are the wrong size, broken, out of date, or are not actually ordered. The market for managing the return of goods is growing as the pace of e-commerce retail sales accelerates. While the historical rate for returning merchandise is about $5 \%$, some estimates suggest that online-driven products realize return rate in excess of $30 \%$, which is a logistics headache. Although the market for forward logistics is much larger than that of reverse logistics, the market opportunity for reverse logistics is getting bigger. B2B e-commerce growth is further fueling the need to manage the return of goods purchased on-line.

\subsection{Growing competition in the market and consolidation}

A relatively small number of delivery companies currently deliver the vast majority of goods purchased online to customers' homes in the US. This means that at present many retailers are using the same delivery companies to perform home deliveries. As a result, a high degree of consolidation takes place within these delivery companies' operations, helping to provide relatively high delivery densities. However, as more companies begin to offer home delivery services, this could result in lower delivery densities and an increase in delivery costs and vehicle trip generation. There are currently few delivery companies working together in order to improve the consolidation of e-commerce goods. However, this may become increasingly necessary for economic, traffic and environmental reasons if the number of delivery companies offering home delivery grows significantly in future. A fundamental premise of the home delivery business is that to operate profitably, a delivery company needs to have a certain density of goods to deliver within a given region. As density increases, the productivity and profits of delivery companies will also rise.

\section{Conclusions}

In this paper, we have investigated key issues associated with home delivery of e-commerce goods, and presented potential strategies that may lead to more efficient and reliable home delivery 
systems. Home delivery services for e-commerce goods stimulate greater complexity in the traditional freight supply chain management. The delivery problem when the customer is not attended at the time of delivery is the most critical factor for the success of home delivery operations. Many home deliveries involve customer demands that are extremely time-sensitive and unsuitable for mixing with other home deliveries at the same time, leading to little opportunity for consolidation. These features cause higher operating costs for carriers and lower customer satisfactions for home delivery services. Increased demands for home delivery services could also lead to a significant increase of delivery vehicle trip generation in urban areas, raising social and environmental issues such as traffic congestion and air pollution. Potential strategies for their solution include the establishment of reception boxes or local pickup points to reduce redundant delivery attempts in home delivery and the possibility of the increase in the lead-time between ordering and delivery. The increasing importance of local operations, reverse logistics and consolidation for smaller goods and between home delivery companies, were additionally addressed. Advances in home delivery have the potential to expedite the growth of e-commerce as well as to create sustainable urban freight transportation systems.

However, the difficulty in implementing a new model is resistance to change. For the development of better home delivery models, it is worthy of noting that customer's needs and preferences for new services should be primarily recognized. There are still many other types of home deliveries that have not explicitly been examined in this research, such as consumer-toconsumer e-commerce goods home delivery or same-day home delivery. There is also still a lack of evidence about whether the overall impacts of replacing customer's shopping trips with home delivery operations are positive or negative. Future research should delve deeper into these domains.

\section{References}

Airborne Express. 2003. SEC Filings: Annual and quarterly reports. Available at www.airborne. com.

Bureau of Transportation Statistics. 1999. 1997 Commodity flow survey. US Department of Transportation. Available at www.bts.gov/ntda/nts/NTS1999/

Cairns, S. 1996. Delivery alternatives: Successes and failure of home delivery services for food shopping, Transport Policy, 3(4), 155-176.

Campbell, A.M. and M. Savelsbergh. 2002. Decision support for direct grocery initiatives. Working Paper, University of Iowa.

Census Bureau. 2003. Estimated quarterly US retail e-commerce sales, US Department of Commerce. Available at www.census.gov/estats. 
Eno Transportation Foundation. 2002. Transportation in America: A statistical analysis of transportation in the United States. Washington. DC.

Federal Express. 2003. SEC Filings: Annual and quarterly reports. Available at fdx.client.shareholder.com/edgar.cfm.

Golob, T. F. and A. C. Regan. 2001. "Impacts of information technology on personal travel and commercial vehicle operations: Research challenges and opportunities, Transportation Research, 9C, 87-121.

Golob, T.F and A.C. Regan. 2002. Trucking industry demand for information technologies: A multivariate discrete choice model Transportation Research, 10C, 205-228.

Gould, J. 1998. Driven to shop? Role of transportation in future home shopping, Transportation Research Record 1617, 149-156.

Gould, J. and T. Golob. 1998. Will electronic home shipping reduce travel?, Access, 12, 26-31.

Gulati, R. and J. Garino. 2000. Get the right mix of bricks \& clicks, Harvard Business Review, 78(3), 107-115.

Johnson, C.A., K. Delhagen and E.H. Yuen. 2003. US eCommerce Overview: 2003 To 2008, Forrester Research, Cambridge, MA.

Lyons, G. 2002. Internet: Investigating new technology‘s evolving role, nature and effects on transport, Transport Policy, 9, 335-346.

Morlok, E. K., B. F. Nitzberg, and K. Balasubramaniam. 2002. The parcel service industry in the US: Its size and role in commerce, department of systems engineering, School of engineering and applied science, University of Pennsylvania, Philadelphia.

Nagarajan, A., E. Canessa, W. Mitchell, and C. C. White III. 2000. "E-commerce and the changing terms of competition in the trucking industry: A study of firm level responses to changing industry structure, Presented at the Berkeley Roundtable on the International Economy (BRIE), University of California at Berkeley, CA, April 2829, 2000.

Nemoto, T., J. Visser and R. Yoshimoto. 2001. Impacts of information and communication technology on urban logistics system, Presented at the OECD/ECMT Joint Seminar on The Impact of E-commerce on Transport: Session 4 Transport and Local Distribution, Paris, France, June 5-6, 2001.

United Parcel Service (2003) SEC Filings: Annual and Quarterly Reports, 1994 to 2002. Available at www.shareholder.com/ups/edgar.cfm.

United States Postal Service (2003) USPS 2002 Annual Report. Available at www.usps.com /history/anrpt02/

$\mathrm{http}: / / \mathrm{www}$.dynamex.com

http://www.officedepot.com 